J. Theoret. Biol. (1969) 23, 425-440

\title{
Control of Cellular Processes by the Coupling of Resonant Energy to Hydrogen Transfer Reactions
}

\author{
I. Application to Enzyme Mechanisms
}

\author{
NEWTON RESSLER $†$ \\ The Departments of Pathology and Biological Chemistry, \\ The University of Michigan Medical Center, \\ Ann Arbor, Michigan 48104, U.S.A.
}

(Received 21 October 1968)

\begin{abstract}
A hypothesis is presented that electronic and vibrational resonant energy states are important in biochemical reactions due to their coupling to hydrogen transfer reactions. The hypothesis is based upon (1) the involvement of hydrogen in most biochemical reactions, (2) the unique ability of hydrogen to act as an energy trap for resonant energy and (3) demonstrations of the coupling of resonant energy to hydrogen transfers in various systems. The dependence of the transfer of resonant energy upon the overlapping of energy levels, and upon molecular geometry, can be related to the sequence or coordination of biochemical reactions, and to the requirement of structural integrity of cellular components for many biochemical processes. The reversibility of the hydrogen transfers may provide a means for the storage and subsequent liberation of the resonant energy. Some characteristics of resonant energy states, and the ability of hydrogen to act as a trap for resonant energy are discussed. The coupling of resonant energy to hydrogen transfers is related to mechanisms of enzyme action. Possible means of confirming this hypothesis and a comparison of it to some other related processes are presented.
\end{abstract}

\section{Introduction}

It is becoming apparent from various investigations that the resonant transfer of energy, after excitation, is a common phenomena in proteins, nucleic acids, and other biochemical constituents (Guéron \& Shulman, 1968; Perlman, Van Zyl \& Edelhoch, 1968). A number of observations suggest that resonant energy states are important in biochemical processes. The most direct evidence for resonant energy transfer, in vivo, has been obtained during photosynthesis, and discussed by Rabinowitch (1963). The intimate relationship between some

$\dagger$ Present address: Departments of Pathology and Biological Chemistry, University of Illinois Medical Center, Chicago, Ill. 60612, U.S.A. 
cases of bioluminescence and mitochondrial respiration (Sadana \& McElroy, 1957) suggests the possibility that the generation of excitation energy is not restricted to bioluminescence, but may also be involved in mitochondrial respiration and other processes. The long-term delayed light emission of photosynthesizing plants appears to be best understood in terms of a re-excitation of chlorophyl by stored energy (Bertsch, Azzi \& Davidson, 1967) and is consistent with resonant energy transfer to or from sites where energy can be stored by an endergonic photochemical reaction, and liberated by its reversal.

Various enzymes have been shown to require or to be activated by excitation energy. The experiments of Bassham (1968), for example, indicate that the activity of fructose 1,6-diphosphatase in Chlorella pyrenoidosa, both in vivo and in isolated chloroplasts, is greatly enhanced in the presence of light, which may act as a regulatory mechanism. Orlando (1968) has shown that light can induce a transhydrogenation reaction similar to the transhydrogenase reaction in mitochondria, i.e. the direct transfer of hydrogen from NADH to NADP without an exchange with the medium. Enzyme systems in vivo may be rapidly activated or inactivated as a unit, and in a manner not directly related to the apparent concentrations of substrates, cofactors, or effectors (Helmreich \& Cori, 1965). The activation of enzyme systems by means of the transfer of resonant energy between adjacent enzymes would provide one means of accounting for such phenomena. The dependence of the transfer of resonant energy upon the overlapping of energy levels and upon molecular geometry could be related to co-ordinated or specific sequences of reactions and to the effect of the integrity of the structure of cellular components upon enzyme activity.

All investigators do not agree that resonant energy states are important in biochemical processes. Some of the reasons for reservations about this possibility are: (1) ATP cannot generally supply sufficient energy to form excited electronic states; (2) the activation energy required for most enzyme reactions is considerably less than is involved in excited electronic states; (3) the lifetime of excited resonant energy states is a small fraction of the time required for most biochemical processes (Davydov, 1962); and (4) the mechanism by means of which resonant energy could induce specific biochemical reactions is not generally clear.

Additional considerations suggest, however, that an important role for resonant energy states is still not impossible. It has become recognized that ATP is not the exclusive source of utilizable energy (Pullman \& Schatz, 1967), and that the cellular production of considerably more energy than can be liberated by ATP may not be restricted to bioluminescence (Vorhaben \& Steele, 1967). On the other hand, spectral studies have demonstrated that 
vibrational energy can also result in excited resonant energy states, which have many of the characteristics of electronic excited resonant energy states (Davydov, 1962; Polonsky, 1964). Vibrational or electronic resonant energy is generally coupled to hydrogen transfer reactions when possible (Walrafen, 1967; Poland \& Scheraga, 1965), due to the efficiency of hydrogen in acting as an energy trap. Most biochemical reactions involve hydrogen transfers (Rose, 1966). It is, therefore, possible that the coupling of either electronic or vibrational excited resonant energy, depending upon the energy requirements, to hydrogen transfers, may constitute a biological role for excited resonant energy which is not excluded by the reservations described.

Consequently, a consideration of possible relations between enzyme activity and the trapping of resonant excitation energy by hydrogen transfer reactions may be of interest. The present communication consists of a discussion of: (1) some characteristics of excited resonant energy states; (2) the coupling of resonant energy to hydrogen transfer reactions; (3) the possible involvement of this coupling in enzyme reactions; (4) possible confirmation of this involvement; and (5) a comparison of this mechanism to some other, related phenomena.

\section{Characteristics of Excited Resonant Energy States}

Excited electronic and vibrational resonant energy, shared collectively by a number of molecules, does not require orbital overlaps. The energy can be shared or transferred between molecules not in direct contact, over relatively long ranges (i.e. 10 to $50 \AA$ ). When electronically excited molecules have similar energy levels, non-radiative interactions, involving a coupling of polar transitions, may occur between them. The interacting excitation energy, referred to as an exciton, has been characterized in various systems and discussed by Förster (1960), Kasha (1959) and Davydov (1962). The transfer of excitation energy between molecules depends upon the energy of interaction between the excited and unexcited configurations. In the case of two like molecules, $A$ and $A^{\prime}$, in excited $A^{*}$ and $A^{\prime *}$ and unexcited, $A$ and $A^{\prime}$ states, the interaction is between the configurations $A^{*} A^{\prime}$ and $A^{\prime *}$. As has been discussed by Förster (1960), the interaction energy, $\mu$, can be described by the expression:

$$
\mu=\iint \psi_{\mathrm{A}^{\circ}}(x) \psi_{\mathrm{A}^{\prime}}\left(x^{\prime}\right) H \psi_{\mathrm{A}}(x) \psi_{\mathrm{A}^{\prime} \cdot}\left(x^{\prime}\right) \mathrm{d} x \mathrm{~d} x^{\prime}
$$

in which $x$ and $x^{\prime}$ are the electronic co-ordinates, $\psi_{\mathrm{A}}$, etc., are the molecular electronic cigenfunctions, and $H\left(x, x^{\prime}\right)$ the interaction operator.

If the magnitude of this interaction energy is sufficiently large, the rate of transfer of the exciton is so great that an entire aggregate of molecules can be considered to be sharing the excitation at the same time. The time of 
transfer is less than the vibrational periods, and the equilibrium internuclear positions are intermediate between the ground and excited states. Excitons in this case are referred to as free or delocalized excitons.

If the interaction energy is relatively low, i.e. weak coupling, the rate of exciton transfer is reduced and the time required for the transfer between molecules may be considerably more than the period of nuclear vibration. Consequently, the migration of the exciton, as it spreads, is accompanied by a displacement of the molecules into new equilibrium positions. The excitons in this case are referred to as localized.

The case of very weak coupling has been described by Förster (1960). It involves a quantum mechanical vibrational-relaxation mechanism of dipoledipole interaction, with an $r^{-6}$ dependence. This type of transfer is relatively slow, with rates in the $10^{9}$ to $10^{6} \mathrm{sec}^{-1}$ range.

The basic mechanism of excitation transfer is the same despite the strength of the coupling and differences in coupling reflect different arrangements for energy transfer under different conditions. The differences in coupling depend upon the magnitude of the interaction energy compared to the width of the electronic or vibronic bands, and the interaction energy in the case of very weak coupling is not necessarily small on an absolute scale.

The probability of transfer of resonant energy depends upon the overlapping of the emission band of the energy donor with the absorption band of the energy acceptor (Rabinowitch, 1963). The direction of migration is from molecules of slightly higher energy levels to molecules of slightly lower energy levels. The migration of resonant energy in the reverse direction, i.e. to higher excited states, is considerably less probable, since it requires an extraneous source of energy. The tendency of resonant energy to migrate along molecules in a definite direction, i.e. in the order of the most gradual decrease of excited energy levels, is of possible significance in the ordering of biochemical reactions.

Resonant energy can be reduced or lost by such processes as radiation, utilization for chemical reactions, or conversion into vibrational energy or heat. A single exciton may be converted into two or more excitons of lower energy and a localized exciton may be formed from a free one, due to a conversion of some of its energy into vibrational energy (Davydov, 1962).

Many of the characteristics of electronic excitons also apply to vibrationally excited resonant energy (Davydov, 1962), referred to as vibrational excitons. The latter also vary in their extent of localization or delocalization, requirement of energy level matching for transfer etc. The interactions of electronic with vibrational excitons have recently been discussed by Takeno (1967), and by Philpott (1967). Electronic excitons may actively capture or liberate vibrational excitons in various cases. The activation energy for a chemical 
reaction can be supplied by a vibrational exciton liberated from an electronic exciton, when only a portion of the energy of the latter is required.

Both electronic (Nicolis \& Rice, 1967) and vibrational (Chantry \& Gebbie, 1965) resonant energy transfers may occur in liquids. Resonant energy transfer processes can be considerably more efficient, however, in solid systems. The specific structures of proteins and nucleic acids, or the organization in structures such as mitochondria are relatively favorable for resonant energy states (Magee, 1960). There is a high degree of rigidity, and restriction of a number of vibrational degrees of freedom in these cases (Edelhoch \& Steiner, 1964).

It should be noted that the lifetimes of excited resonant energy states are generally less than $10^{-6} \mathrm{sec}$, and they cannot be regarded as a means of energy storage. Resonant energy may be stored indirectly, by its use for an endergonic reaction. The energy can be made available later, by the subsequent exergonic conversion of the reaction product to another substance of a lower potential energy. If the initial reaction involves a hydrogen transfer which acts as an energy sink, the resonant energy can be utilized with a high degree of efficiency.

\section{The Induction of Hydrogen Transfers by Excited Resonant Energy}

Molecular orbitals for the combined $\mathrm{NH}_{3}$ and $\mathrm{HCl}$ system have recently been calculated by Clementi (1967). $\mathrm{As} \mathrm{NH}_{3}$ and $\mathrm{HCl}$ approach each other, there is at first a mutual polarization which results in a net gain of energy, even at the relatively large distance of $15 \AA$. The proton of $\mathrm{HCl}$ is transferred to $\mathrm{NH}_{3}$ and an $\mathrm{NH}_{4}^{+} \mathrm{Cl}^{-}$ion pair is formed. The equilibrium position of $\mathrm{N}$ is off the center of the mass, within the sphere of the four protons. The type of some of the energy diagrams obtained is shown in Fig. 1.

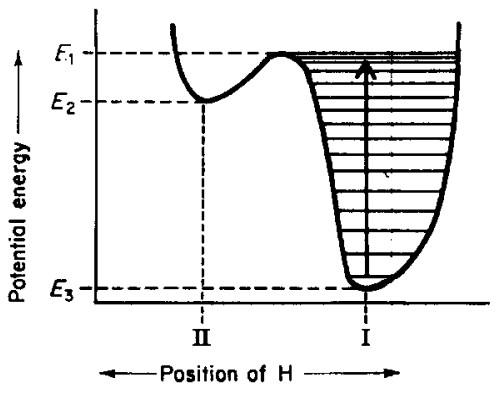

Fig. 1. Variation of activation energy for proton transfer with $\mathrm{N}-\mathrm{Cl}$ internuclear distance. As the $\mathrm{N}-\mathrm{Cl}$ distance decreases the activation energy also decreases. This is true for the transfer of a proton either from position I to $I\left(E_{a}\right)$, or from II to I $\left(E_{a}^{\prime}\right)$. 
The transfer of the proton from $\mathrm{HCl}$ to $\mathrm{NH}_{3}$ involves the passage over an energy barrier, $\Delta E . \Delta E$ decreases until it reaches zero as $\mathrm{N}$ and $\mathrm{Cl}$ approach closer to each other, due to the gain in energy from the $\mathrm{NH}_{3}$ and $\mathrm{HCl}$ interactions. The decrease in $\Delta E$ with the closer approach of the two groups between which the hydrogen is exchanged may be related to the reduction of activation energies in enzyme or in other biological hydrogen transfer reactions, as will be described. The precise shape of energy diagrams for hydrogen transfer reactions depends, in any particular instance, upon spatial or geometrical arrangements and charges or polarities of other neighboring groups (Bratoz \& Hadzi, 1957). In general, however, the gain in interaction energy, with the mutual approach of two groups that hydrogen is transferred between can be expected to result in a reduction of the energy barrier $\Delta E$ (Clementi, 1967).

Excitation energy can induce hydrogen transfer reactions by means of providing sufficient energy for the hydrogen to pass over the energy barrier, $\Delta E$. This could be accomplished by either electronic or vibrational excitations. In the former case, photodissociation by such mechanisms as pre-dissociation or electronic excitation to a repulsive state have been discussed by Herzberg (1939). Spectral evidence that electronic excitations can lead to proton transfer reactions in organic molecules has been presented by Weller (1956) and by Mataga, Kaifu \& Koizumi (1955). Voevodskii \& Molin (1962) have discussed reasons why the dissociation of an $\mathrm{R}-\mathrm{H}$ bond and the formation of hydrogen atoms is commonly regarded as the primary act of radical formation after the irradiation of solids. The ability of bonds containing hydrogen to act as energy sinks for electronic excitation energy depends upon the use of this excitation energy for the increase of the vibrational energy of hydrogen. Lin \& Bersohn (1968) have presented evidence that the high frequency vibrational stretching modes of hydrogen are the primary acceptors of electronic excitation energy.

Various experiments demonstrate that resonant transfer of the electronic excitations can be coupled to hydrogen transfers. Weber \& Rosenheck (1964) found that hydrogen transfer reactions are four to five times more effective than the total of all other processes in quenching the fluorescence of tyrosine copolymers [i.e. in accepting the excitation energy (Voevodskii \& Molin, 1962)]. Poland \& Scheraga (1965) have suggested, on the basis of intensity and polarization of fluorescence studies, that excitons in proteins transfer their energy to hydrogen-bonded groups, which act as energy sinks. Yomosa (1964) interpreted optical absorption, thermal excitation, and conduction properties of hydrogen-bonded polypeptides in terms of exciton bands. He concluded that vibrational coupling with the proton of the hydrogen bond is involved in the transfer of the triplet exciton. Ashraf El-Bayoumi \& Kasha 
(1961) have also presented spectral evidence that a hydrogen-bonded complex can act as an energy sink for resonant excitation energy.

The inhibition of a hydrogen transfer by an energy barrier can also be reduced by the resonant transfer of vibrational energy to the vibrational modes of the hydrogen containing bond. (Chemical reactions generally depend upon the gain of sufficient vibrational energy in bonds which become dissociated.) As hydrogen's vibrational energy state approaches the level of $E_{1}$ in Fig. 2 (indicated by the vertical arrow), the inhibition of the transfer
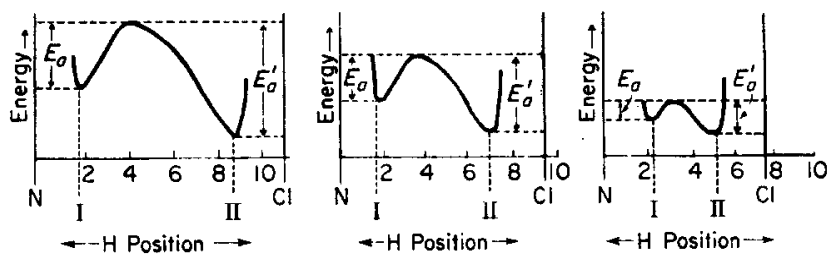

Fra. 2. Use of hydrogen transfer for energy storage or liberation. Energy is liberated by a transfer of a hydrogen from position II to I. Energy storage is accomplished by a hydrogen transfer from position I to II. The increase in vibrational energy to higher levels (indicated by the arrow over position I) facilitates passage over the energy barrier.

of hydrogen from position I to II by the energy barrier decreases. The induction of a hydrogen transfer depends upon the extensive ability of the vibrational modes of hydrogen to act as energy acceptors. Lambert (1967) in a review of the transfer of vibrational energy, has pointed out that the resonant transfer of vibrational energy to groups containing hydrogen atoms has an unusually high efficiency. The reason for this can be understood in terms of the expression derived by Tanczos (1956) for resonant energy exchange. When two molecules, $a$ and $b$, approach each other, the probability of intermolecular resonant energy exchanges $P(a, b)$ is:

$$
P(a, b)=P_{0}(a) P_{0}(b) V^{2}(a) V^{2}(b) \frac{64 \pi^{2} \mu k T}{\alpha^{2} h^{2}} \exp -\left(\phi_{0} / k T\right) .
$$

$P_{0}(a)$ and $P_{0}(b)$ are factors which depend upon geometrical orientations, $V^{2}(a)$ and $V^{2}(b)$ are vibrational factors depending upon the quantum changes involved; $\mu$ is the reduced mass of the molecules, $\alpha$ is the intermolecular force constant, and $\phi_{0}$ is the minimum value of the intermolecular potential function. The equation predicts that the probability of transfer in hydrogen-containing groups should be comparatively high, since hydrogen atoms have a relatively low mass. The large frequency amplitudes, due to the small mass of hydrogen, results in a large increase in the vibrational factors. A comparison of various molecules by means of calculations based upon 
available data confirms that the transfer efficiency of vibrational energy involving hydrogen-containing groups is high (Lambert, 1967). Similar considerations may apply to both protons and hydride ions, since their masses are very nearly the same. The ability of protons to act as a sink for either electronic or vibrational energy is also related to the high rate of proton transfer reactions, which may be as great as $10^{-13} \mathrm{sec}^{-1}$ (Eigen \& De Maeyer, 1958). It is surprising, perhaps that the rate of proton transfer reactions is sometimes greater than the rate of electron transfer processes (Eigen \& Hammes, 1963).

Spectroscopic studies have demonstrated that hydrogen transfers may be directly coupled to the transfer of vibrationally excited resonant energy. In an investigation of intermolecular vibrational coupling, Walrafen (1967) obtained argon-ion laser and unpolarized mercury-excited Ramon and infrared spectra, of aqueous selenious acid solutions at various concentrations. A persistent, characteristic out of phase coupled component at all concentrations indicated that the predominant coupling mechanism involved the hydrogen bonds of water molecules (which were between the coupled $\mathrm{H}_{2} \mathrm{SeO}_{4}$ molecules). Jakobsen, Mikawa \& Brasch (1967) have obtained spectra of hydrogen-bonded $n$-decanol which indicate that the width of the $\mathrm{OH}$ stretching vibration in the hydrogen bond results from first-order coupling with the other, adjacent, hydrogen bonds. The interaction involves the formation of tautomers from coupled in phase proton-transfer reactions. Other lower frequency hydrogen bond modes may also contribute to the band width. The results demonstrate that hydrogen transfer reactions can be induced by vibrational resonant energy states.

Potential energy diagrams for hydrogen transfers are not necessarily symmetrical. In Fig. 2, for example, if a hydrogen is transferred from position I to position II, energy equal to $E_{1}-E_{3}$ is necessary in order for the reaction to proceed, and $E_{1}-E_{2}$ is liberated. Resonant energy could be stored by such a hydrogen transfer, since, at a later time the hydrogen could return from position II to I, with the liberation of energy equal to $E_{1}-E_{3}$. The liberation of this energy might be coupled to a subsequent, endergonic reaction, such as the formation of ATP, or it might involve the liberation of additional resonant energy. Bioluminescence, when preceded by resonant energy transfer before the light emission, may be analogous to the latter process.

In conclusion, evidence has been discussed that hydrogen transfers are induced by vibrationally or electronically excited resonant energy. This is due to the ability of vibrational modes of hydrogen to accept, or act as efficient traps for, resonant energy. The hydrogen transfer, in conjunction with other coupled group transfers, may result in the storage of resonant energy and 
its subsequent liberation. Resonant energy could, of course, have other biological roles, and hydrogen transfers can occur by tunneling through, rather than passing over, energy barriers. These possibilities are discussed below.

\section{Enzyme Mechanisms}

Rose (1966), in a recent review of enzyme mechanisms, has indicated that while it is generally recognized that acid-base catalysis and proton transfers play a role in enzyme reactions, relatively little attention has been paid to the relation between the proton transfer and the reduction of activation energy by the enzyme. Some aspects of this problem have been discussed by the present author (1958). The possible mechanism considered depends upon reacting groups of the enzyme being positioned adjacent to groups of the substrate with which they interact, due to the enzyme's stereospecificity. If a proton is to be transferred from a group $A$ on the substrate to a group B on the enzyme, and if the binding in the enzyme-substrate complex is such that groups A and B are positioned very closely together, then the gain in interaction energy can result in a decrease in the activation energy for the proton transfer as discussed above (Clementi, 1967). The dependence of the reduction of the activation energy upon the energy gained by the enzymesubstration interaction could account for the critical effects upon enzyme activity of the structure of the substrate and tertiary structure of the enzyme (i.e. in positioning reacting groups).

When a proton is transferred from substrate to enzyme (or coenzyme), the substrate is left with a negative charge, and the enzyme with a positive charge. These resultant charges tend to induce either a second proton transfer in the opposite direction from the first one, or the transfer of a negatively charged group, such as $(\mathrm{OH})^{-},(\mathrm{SH})^{-}$, a hydride anion or electron, in the same direction. The tendency to neutralize the charges resulting from the first proton transfer can thus lower the activation energy for a subsequent or accompanying transfer (Lowdin, 1964). In some cases a proton transfer may induce a subsequent reaction involving a hydroxyl group of water (although it is possible that, in these cases, the water molecule is bound in a favorable position).

If the proton and anion (or two opposite proton) transfers occur simultaneously, the mechanism would be a concerted one, as described by Swain \& Brown (1952). It is not necessary to make this assumption, however, since the first transfer would still reduce the activation energy for the second one, if they occur sequentially. Such a mechanism for the reduction of activation energy and for proton transfer through hydrogen bonds is consistent with the fact that there is often a direct transfer of hydrogen between the enzyme and 
substrate (Rose, 1966), i.e. the proton (and negative group) do not usually become free. Exchanges with the medium may occur in other steps. Although the proton transfer can be very rapid, the hydrogen bond may exist for a longer period of time.

The extent to which activation energy may be decreased by an enzyme in the manner described has, of course, not been determined. The mechanism can serve for the present purposes, as one example in which the necessary activation energy could be supplied by resonant energy. The activation of an enzyme or enzyme system could then be related to the availability of resonant energy for the transfer of the proton, hydride anion, or hydrogen atom involved in the reaction (as discussed in section 3).

\section{Confirmation of the Activation of Enzymes by the Transfer of Resonant Energy}

Some experimental observations have been reported which suggest that enzymes can be activated by resonant energy. Reference has been made to the demonstrated requirement of some enzymes for excitation energy, and to demonstrations of the transfer of resonant energy in proteins. Although the transfer of resonant excitation energy between particular protein groups can be experimentally difficult to prove, various results suggest that resonant energy can be transferred between tyrosine or tyrosinate and tryptophan residues (Perlman, Van Zyl \& Edelhoch, 1968). Resonant energy transfer from the tryptophan groups of proteins to various acceptors have been demonstrated (Weber \& Teale, 1959). In NADH, resonant energy is transferred from the adenine to the nicotinamide portions of the molecule, and in NADH bound to an enzyme such as lactic dehydrogenase, resonant energy may be transferred to the tryptophan residues and then to the nicotinamide portion of the coenzyme (Velick, 1961). In the alcohol and lactic dehydrogenase reactions, hydrogen may be transferred in the same order, i.e. from the substrate to a tryptophan residue on the enzyme, and then to the nicotinamide moiety of NAD (Schellenberg, 1967). Reference has also been made to the light induced, direct transfer of hydrogen from NADH to NADP (Orlando, 1968).

The transfer of both resonant excitation energy and hydrogen, between the same constituents and in the same sequence, and the ability of the excitation energy to induce hydrogen transfers, is consistent with the present hypothesis. Similar considerations may apply to the relation between resonant energy and hydrogen transfers in flavin nucleotide containing enzymes (McCormick, Koster \& Veeger, 1967).

More direct evidence for the present hypothesis might be obtained by testing predictions that can be made on the basis of this mechanism. If 
hydrogen transfers and resonant energy are coupled, then resonant energy would occur as pulses corresponding to each hydrogen transfer, rather than in a continuous form. It could thus be predicted that enzymes can be activated by irradiations, if the radiation energy is absorbed by the hydrogen which is transferred in the enzyme reaction, is pulsed at a rate corresponding to the turnover number of the enzyme, and has a suitable wave length.

The relations of such activation to wave length or pulse rate of irradiations and to temperature could help to elucidate the mechanism of activation detected. If the pulsed irradiation, at a given wave length, supplies sufficient energy for the passage of hydrogen over the energy barrier, then thermal energy or molecular collisions are not necessary, and the activity of the enzyme will not decrease with decreasing temperatures (Lin \& Bersohn, 1968). It has been observed in this regard that the light-induced reduction of flavins is not significantly dependent upon the temperature (McCormick et al., 1967).

If a large proportion of the pulsed irradiation energy is used to supply the activation energy for the reaction, then the lowest frequency of irradiating light which can activate the enzyme should decrease as the temperature increases. This is because higher vibrational energy levels will be occupied (in the bond containing the hydrogen to be transferred) at higher temperatures. Less light energy would then be necessary in order to reach the maximum of the energy barrier. (The original vibrational states may, of course, depend upon a Boltzmann distribution, and the decrease in enzyme activation with decreasing frequency may not be sharp.) The prediction that enzyme activation by pulsed irradiations will depend upon the number of pulses per second in relation to the turnover number of the enzyme can also be investigated. It is possible that pulsed irradiations could be experimentally correlated with hydrogen transfers, as well as with enzyme activity, by determining the effects of the pulsed irradiations upon spectrophotometric difference spectra (Weber \& Teale, 1965) or upon proton magnetic resonance spectra.

(The inactivation of enzymes which is often produced by ultraviolet irradiation may be related to light which is of a frequency not suitable for enzyme activation or which is continuous rather than pulsed. Inactivation does not appear to be due to absorption and retention of the radiant energy per se (Yeargers \& Augenstein, 1968), but to specific, environmentally dependent reactions. With continuous irradiation, the energy absorbed after a hydrogen transfer, but before another hydrogen is in an appropriate position to absorb more energy, may result in the migration of energy into channels where inactivation reactions can occur).

In addition to studies of the activation of enzymes by the direct absorption by hydrogen of pulsed excitation energy, investigations of the transfer of 
resonant energy to this hydrogen can also be investigated. Various chromophores, which will absorb and emit light at a variety of wave lengths are available for conjugation with enzymes at different distances from the active site (Galley \& Stryer, 1968). It may thus be possible to correlate the activation of an enzyme to the transfer of resonant energy which had initially been absorbed by a conjugated chromophore. Predictions of the effect of the distance of transfer (between the chromophore and hydrogen) and of the frequency of the pulsed irradiations could be made as described above. The effects of resonant energy transfer from one enzyme to another, in an enzyme system could be investigated by the activation of one enzyme, due to the absorption of pulsed irradiations by a chromophore on another, adjacent enzyme. [The activity of a number of enzymes is known to be affected by the presence of other enzymes in the medium (Scrutton \& Utter, 1968).]

\section{Alternative Possibilities to the Resonant Energy-coupling Hypothesis}

Comparisons to other, related processes may facilitate an evaluation of the hypothesis being considered. In order to remain within the scope of this communication, only selected aspects of a limited number of phenomena are considered.

Semiconduction has been proposed as a means for the transfer of charge which may occur in various biochemical processes. It requires orbital overlap between molecules. In molecular aggregates, the exponential temperature factor depends upon the energy gap from the ground states to a conduction band. Kasha (1962), in a discussion of this mechanism, questioned whether or not there is sufficient orbital overlap between different molecules in an aggregate for a conduction band to develop between them. He has suggested that the similarity of conduction phenomena in inorganic materials and in biochemical molecular aggregates may be more apparent than real. These points can only be clarified by further investigations.

They are obviated in the case of the transfer of resonant energy since contact between molecules is not necessary. In the hypothesis being considered, the transfer of hydrogen between two closely positioned groups in an enzyme-substrate complex does require orbital overlap. Resonant energy, which induces this hydrogen transfer can, however, be transferred to the hydrogen without orbital overlap or molecular contact.

Another alternative to the coupling of resonant energy to hydrogen transfers is the induction by resonant energy of electron transfers. It would appear feasible that the induction of electron as well as proton transfers by resonant energy could be important. As Reynolds \& Lumry (1966) have pointed out, electron and proton transfers may be difficult to distinguish. The transfer of 
two hydrogen atoms, a proton and a hydride ion, or two protons and two electrons could have the same net result. Whether or not the transfer involves a proton, a hydrogen atom, or a hydride ion is not critical since the hydrogen's mass is essentially the same and it still acts as a trap for resonant energy. As explained above, it is not necessary to assume that free protons are liberated into or taken up by the medium in enzyme reactions. Hydrogen rather than electron transfers have been considered as primary in this communication because hydrogen is involved in a large majority of biochemical reactions, and because of the unique ability of hydrogen to act as an energy trap for excitation energy.

In Chromatium, the induction of the oxidation of cytochrome by short pulses of light at low temperatures has been explained by De Vault, Parkes \& Chance (1967), on the basis of electron tunneling. The requirement for energy level matching also provides the opportunity for an energy switch mechanism. Since the probability of tunneling increases exponentially with decreasing mass, the probability of tunneling by electrons is considerably greater than that by protons; the former could be an important biochemical process. Various aspects of electron tunneling have been discussed by Reynolds \& Lumry (1966).

Besides passing over energy barriers, proton transfers can also occur by tunneling through the energy barriers (Lunell \& Sperber, 1967; Rein \& Harris, 1966). Some of the factors which influence proton tunneling have been discussed by Lowdin (1964). The probability of tunneling depends upon the shape of the energy barrier, and decreases exponentially as the barrier's height and width increase. Proton tunneling in the usual sense, does not occur if the transfer must be to a higher potential energy level on the other side of the barrier. Lowdin has calculated the probability that mutations could be due to tunneling. Over the time scale involved in mutations, the results suggested that this mechanism could be involved. He has cautioned, however, that some of the factors which greatly influence tunneling probabilities can only be approximated at the present time. The spontaneous nature of the tunneling process and its relative restriction to certain types of potential energy curves are difficult to relate to the rapid activation or inactivation of enzyme systems as a unit or to reactions involving appreciable energy storage. The possibility cannot, of course, be ruled out that proton tunneling occurs and has an important role in some processes.

Charge fluctuations or dispersion forces should also be considered in the present context. Due to the uncertainty principle, fluctuations in electronic charge result in repulsive and, preferentially, in attractive forces between neutral molecules. As Kirkwood \& Shumaker (1952) have described, attractive forces also result from the fluctuation of protons among basic sites. The 
proton fluctuations could be due to Brownian motion, or to co-ordinated transfers across hydrogen bonds. The intermolecular interactions are considered to take place through a fluctuating electromagnetic field, which has an influence upon chemical reactions. Intermolecular charge fluctuations depend upon the similarity of interacting molecules, and can relate to biological specificity. Breuer (1968) has illustrated how proton dispersions may lead to co-operative interactions at different sites.

Charge fluctuations and resonant energy processes are not entirely different, and the interactions can occur by a resonant mechanism (Jehle, Parke \& Salyers, 1964). Co-ordinated fluctuations of both protons and electrons in an electromagnetic field are analogous to the reversible coupling of resonant energy to proton transfers considered in this paper. To the extent that proton fluctuations are unco-ordinated or result from Brownian motion, the process differs from the present hypothesis. Another distinction is that in the present hypothesis, the selective channeling of resonant energy in a particular direction has been related to the controlled induction of the hydrogen transfers involved in enzyme reactions or in energy storage.

Different investigators have proposed that vibrational energy may be important for enzyme activity. Weber (1955) has suggested that the resonant transfer of excited vibrational energy between an enzyme and substrate leads to the activity. Weber's suggestion is consistent with the present hypothesis, in which the activation is related to the induction of the transfer of the hydrogen. Phillipson (1968) has proposed that vibrational excitations, derived from thermal collisions, are transferred in the enzyme to the active site, by a resonant mechanism. This proposal differs from the hypothesis considered here in that the source of resonant energy in the latter case is regarded not as being derived from collisions, but from energy liberated by coupled, exergonic reactions. (The original source of resonant energy is, of course, solar radiation.)

Since enzyme reactions can occur, in vitro, in free solution at room temperature, the necessity of resonant excitation energy for the activation of enzymes should be considered. It is generally observed that the activities of enzymes, in vivo, are not continuous, but co-ordinated with various cellular activities at the appropriate times. As a muscle contracts, for example, the entire glycolytic system may change from an inactive to a highly active state within seconds, and the activity is not directly related to the apparent concentrations of substrates, or effectors (Helmreich \& Cori, 1965). The specific tertiary structures of enzymes and integrity of the cellular architecture can result in a restriction of various vibrational degrees of freedom, and of molecular collisions (Edelhoch \& Steiner, 1964). This provides the opportunity for a selective activation of enzymes in the cell by the channeling of 
resonant energy, although thermal energy is, of course, essentially for various purposes.

\section{Conclasion}

The related phenomena or theories considered above are not mutually exclusive in all respects, and it is not necessary to assume that only one mechanism can be important. In reference to the hypothesis proposed in this communication, the coupling of resonant energy states to hydrogen transfer reactions, due to the ability of hydrogen to act as a highly efficient energy trap, might be regarded as a possible extension of photochemical reactions in original biochemical processes.

The dependence of the transfer of resonant energy upon the matching of energy levels and upon molecular geometry may relate to the determination and co-ordination of reaction sequences and to the effect of cellular structure upon enzyme activities. The possible operation of such relationships is discussed in a subsequent communication.

This work was supported by a grant from the U.S. Public Health Service (HE-10361).

\section{REFERENCES}

Ashraf El-Bayoumi, M. \& Kasha, M. (1961). J. chem. Phys. 34, 2181.

Bassham, J. A. (1968). In abstract, Am. Chem. Soc. 155th national meeting.

Bertsch, W., AzZI, J. R. \& DAVIDson, J. B. (1967). Biochim. biophys. Acta, 143, 129.

Bratoz, S. \& HADZI, D. (1957). J. chem. Phys. 27, 991.

BreUER, M. M. (1968). Nature, Lond. 218, 1076.

Chantry, G. W. \& Gebie, H. A. (1965). Nature, Lond. $208,378$.

Clementi, E. (1967). J. chem. Phys, 46, 3851.

DAVYDOV, A. S. (1962). "Theory of Molecular Excitons". New York: McGraw-Hill.

De Vault, D., Parkes, J. H. \& Chance, B. (1967). Nature, Lond. 215, 642.

EDELHOCH, H. E. \& STEINER, R. F. (1964). In "Electronic Aspects of Biochemistry", p. 7

(B. Pullman, ed.). New York: Academic Press.

EIGEN, M. \& De MAEYer, L. (1958). Proc. R. Soc. A247, 505.

EIGEN, M. \& HAMames, G. G. (1963). In "Advances in Enzymology" (F. F. Nord, ed.). New York: Interscience Publishers.

Förster, T. H. (1960). In "Comparative Effects of Radiation", p. 300 (M. Burton, J. S. Kirby-Smith and J. L. Magee, eds.). New York: John Wiley and Sons.

Galley, W. C. \& Stryer, L. (1968). Proc. natn. Acad. Sci. U.S.A. 60, 108.

Gúron, M. \& Shurman, R. G. (1968). A. Rev. Biochem. 37, 571.

HeLmReich, E. \& CORI, C. F. (1965). In "Advances in Enzyme Regulation" (G. Weber, ed.). New York: Pergamon Press.

Herzberg, G. (1939). "Molecular Spectra and Molecular Structure. I. Diatomic Molecules". New York: Prentice Hall.

Jakobsen, R. J., Mikawa, Y. \& Brasch, J. W. (1967). Nature, Lond. 215, 1071.

Jehle, H., Parke, W. C. \& Salyers, A. (1964). In "Electronic Aspects of Biochemistry", p. 313 (B. Pullman, ed.). New York: Academic Press. 
Kasha, M. (1959). Rev. Modern Phys. 31, 162.

KASHA, M. (1962). In "Horizons in Biochemistry", p. 583 (M. Kasha and B. Pullman, eds.). New York: Academic Press.

KIRKWOOD, J. G. \& ShUMaker, J. B. (1952). Proc. natn. Acad. Sci. U.S.A. $38,863$.

LAmbert, J. D. (1967). Q. Rev. Chem. Soc. 21, 67.

LIN, S. H. \& Bersohn, R. (1968). J. chem. Phys. 48, 2732.

LowDIN, P. (1964). In "Electronic Aspects of Biochemistry", p. 167 (B. Pullman, ed.). New York: Academic Press.

LUNell, S. \& SPERBER, G. (1967). J. chem. Phys. 46, 2119.

MAGEe, J. L. (1960). In "Comparative Effects of Radiation", p. 130 (M. Burton, J. S. KirbySmith and J. L. Magee, eds.). New York: John Wiley and Sons.

Mataga, N., Kaifu, Y. \& KolzUm, M. (1955). Nature, Lond. 175, 731.

McCormick, D. B., Koster, J. F. \& VeEger, C. (1967). Eur. J. Biochm. 2, 387.

Nicours, G. \& RICE, S. A. (1967). J. chem. Phys. 46, 4445.

ORlando, J. A. (1968). Arch. biochem. Biophys. 124, 413.

Perlman, R. L., Van Zyl, A. \& Edelhoch, H. (1968). J. Am. chem. Soc. 90, 2168.

Phillipson, P. E. (1968). J. molec. Biol. 31, 319.

Philpott, M. R. (1967). J. chem. Phys. 47, 2534.

Poland, D. C. \& Scheraga, H. A. (1965). Biopolymers, 3, 369.

Polonsky, J. (1964), In "Electronic Aspects of Biochemistry", p. 481 (B. Pullman, ed.). New York: Academic Press.

Pullman, M. E. \& Schatz, G. (1967). A. Rev. Biochem. 36, 539.

RaBinowrtch, E. (1963). In "Fifth International Symposium of Biochemistry", p. 15 (H. Tamiya, ed.). New York: Pergamon Press.

REIN, R. \& HARRIS, R. E. (1966). J. chem. Phys, 45, 1797.

RessLER, N. (1958). Enzymologia, 19, 310.

REYNOLDS, W. \& LUMRY, R. W. (1966). "Mechanisms of Electron Transfer". New York: Ronald Press.

Rose, I. A. (1966). A. Rev. Biochem. 35, 23.

SAdANA, J. C. \& McElroy, W. D. (1957). Arch. biochem. Biophys. 67, 16.

SCHEllenberg, K. A. (1967). J. biol. Chem. 242, 1815.

Scrutton, M. C. \& UtTer, M. (1968). A. Rev. Biochem. 37, 249.

Swain, C. G. \& Brown, J. F., JR. (1952). J. Am. chem. Soc. 74, 2538.

TAKeno, S. (1967). J. chem. Phys. 46, 2481.

Tanczos, F. I. (1956). J. chem. Phys. 25, 439.

Velick, S. F. (1961). In "Light and Life", p. 108 (W. D. McElroy and B. Glass, eds.). Baltimore: The Johns Hopkins Press.

VoevodskiI, V. V. \& Molin, Y. N. (1962). Radiat. Res. 17, 366.

Vorhaben, J. E. \& SteEle, R. H. (1967). Biochemistry, 6, 1404.

Walrafen, G. E. (1967). J. chem. Phys. 46, 1870.

Weber, G. (1955). Discuss. Faraday Soc. $20,156$.

Weber, G. \& RosenHECK, K. (1964). Biopolymers Symposium 1, 333.

Weber, G. \& Teale, F. W. J. (1959). Discuss. Faraday Soc. 27, 134.

Weber, G. \& Teale, F. W. J. (1965). In "The Proteins", Vol. 3, p. 445 (H. Neurath, ed.). New York: Academic Press.

WELLER, A. (1956). Z. Elektrochem. 60, 1144.

Yeargers, E. \& Augenstein, L. (1968). Biophys. $J .8,500$.

YomosA, S. (1964). Biopolymers Symp. 1, 1. 